\title{
Peripherally inserted central catheters in non-hospitalized cancer patients: 5-year results of a prospective study
}

\author{
Paolo Cotogni - Cristina Barbero • Cristina Garrino • Claudia Degiorgis • \\ Baudolino Mussa • Antonella De Francesco • Mauro Pittiruti
}

Received: 7 March 2014 / Accepted: 4 August 2014 / Published online: 14 August 2014

(C) The Author(s) 2014. This article is published with open access at Springerlink.com

\begin{abstract}
Purpose Few prospective follow-up studies evaluating the use of peripherally inserted central catheters (PICCs) to deliver chemotherapy and/or home parenteral nutrition (HPN) have focused exclusively on oncology outpatients. The aim of this prospective study was to assess the reliability and the safety of PICCs over a 5-year use in non-hospitalized cancer patients requiring long-term intravenous therapies.

Methods Since June 2008, all adult oncology outpatient candidates for PICC insertion were consecutively enrolled and the incidence of catheter-related complications was investigated. The follow-up continued until the PICC removal.

Results Two hundred sixty-nine PICCs in 250 patients $(98 \%$ with solid malignancies) were studied, for a total of 55,293 catheter days (median dwell time 184 days, range 15-1,384). All patients received HPN and $71 \%$ received chemotherapy during the study period. The incidence of catheter-related bloodstream infections (CRBSIs) was low (0.05 per 1,000 catheter days), PICC-related symptomatic thrombosis was
\end{abstract}

P. Cotogni $(\bowtie)$

Anesthesiology and Intensive Care, Unit of Parenteral Nutrition in Oncology, Department of Medicine, S. Giovanni Battista Hospital, University of Turin, Via Giovanni Giolitti 9, 10123 Turin, Italy

e-mail: paolo.cotogni@unito.it

C. Barbero

Department of Cardiovascular and Thoracic Surgery, S. Giovanni

Battista Hospital, University of Turin, Turin, Italy

C. Garrino $\cdot$ C. Degiorgis $\cdot$ B. Mussa

Central Venous Access Team, S. Giovanni Battista Hospital,

University of Turin, Turin, Italy

A. De Francesco

Division of Clinical Nutrition, Department of Medicine, S. Giovanni

Battista Hospital, University of Turin, Turin, Italy

M. Pittiruti

Department of Surgery, Catholic University Hospital, Rome, Italy rare (1.1\%; 0.05 per 1,000 catheter days), and mechanical complications were uncommon $(13.1 \%$; 0.63 per 1,000 catheter days). The overall complication rate was $17.5 \%$ ( 0.85 per 1,000 catheter days) and PICCs were removed because of complications only in $7 \%$ of cases. The main findings of this study were that, if accurately managed, PICCs can be safely used in cancer patients receiving chemotherapy and/or HPN, recording a low incidence of CRBSI, thrombosis, and mechanical complications; a long catheter life span; and a low probability of catheter removal because of complications.

Conclusions Our study suggests that PICCs can be successfully utilized as safe and long-lasting venous access devices in non-hospitalized cancer patients.

Keywords Venous access $\cdot$ Venous access device $\cdot$ Home care $\cdot$ Central venous catheter $\cdot$ Oncology

\section{Introduction}

In non-hospitalized cancer patients, the presence of a venous access device (VAD) is important in the anticancer treatment period for chemotherapy [1] or home parenteral nutrition (HPN) [2], as well as in the advanced phase for palliative care [3]. Choosing the appropriate device for the oncology patient should need a proactive vascular access planning; however, the choice of VAD still largely depends on the therapies' expected duration or on the clinical experience of the provider [1].

Since the 1970s, peripherally inserted central catheters (PICCs) have been available, but only in the last 20 years has their use dramatically increased in several clinical settings [4-16]. The use of PICCs has many advantages over other long-term VADs [17, 18]. First is their ease of insertion due to the placement into a peripheral vein - a safer approach — with the benefit of a central tip location appropriate for any osmolarity and $\mathrm{pH}$ infusions (e.g., chemotherapy drugs, 
hyperosmolar parenteral nutrition (PN) solutions, and longterm antibiotics). PICC placement under ultrasound guidance can be carried out with risks of pneumothorax or hemothorax virtually nonexistent in addition to a low risk of primary malposition $[2,19]$. Moreover, their insertion turns out to be less expensive, as they are usually placed by trained nurses - in an ambulatory setting or bedside - without radiographic or surgical means $[6,17,20,21]$. Also, patients at high risk of hemorrhage needing a central VAD are eligible for PICC insertion with no risk of local bleeding $[1,2]$. Finally, the ease of removal in case of complications offers an adjunctive advantage.

Since 2006, a systematic review of 200 published prospective studies of infection associated with the various types of VADs in adults depicted that PICCs ( $n=2,813 ; 98,702$ days) were at low risk for catheter-related bloodstream infections (CRBSIs) (0.8-1.2 per 1,000 catheter days) in outpatients [13]. Nevertheless, some physicians are still concerned about potential risks associated with the use of PICCs in oncology patients requiring prolonged intravenous therapies due to the reported rates of CRBSIs and thrombosis in earlier PICC experiences [22] and the immunocompromised and prothrombotic tendency of the cancer population [23].

Few prospective follow-up studies evaluating the use of PICCs to deliver chemotherapy and/or HPN have focused exclusively on oncology outpatients. The aim of this study was to assess the reliability and the safety of PICCs over a 5year use in non-hospitalized cancer patients requiring longterm intravenous therapies.

\section{Patients and methods}

Our study was a prospective observational study carried out from June 1, 2008, through May 31, 2013, in a 1,200-bed university hospital. The Ethics Committee approved the study protocol. A written informed consent was obtained from each patient. All adult cancer patients with a PICC inserted during the study period were consecutively enrolled and their followup continued until the PICC removal. Our unit maintains an ongoing prospective database and coordinates the data gathering related to outpatients and is therefore able to determine the true incidence of complications.

Three types of PICCs were used: (1) 4 Fr single-lumen silicone with a valved tip (Groshong PICC; Bard Access Systems, Salt Lake City, UT), (2) 4-5 Fr single-lumen polyurethane (Vascu-PICC; MedComp, Harleysville, PA), and (3) 4-5 Fr single-lumen polyurethane power-injectable (ProPICC; MedComp and Health Line, San Francisco, CA). All PICCs were inserted by specifically trained nurses or physicians of the hospital Central Venous Access Team in a room exclusively dedicated to PICC placement. PICCs were inserted with maximal barrier precautions and skin antisepsis with $2 \%$ chlorhexidine using ultrasound-guided venipuncture of the upper midarm and sutureless devices for securing the catheter. The appropriate central position of the catheter tip (i.e., close to the cavoatrial junction) was consistently verified, either by the intracavitary electrocardiography (EKG) method [24] during the procedure or by chest X-ray after the procedure. According to current guidelines $[25,26]$, routine pharmacological prophylaxis of PICC-related venous thrombosis was not adopted. All patients were screened at least once a year by local ultrasound examination or Doppler technology and echocardiography.

In our hospital, HPN was not routine practice neither in all cancer patients nor the subset receiving chemotherapy. According to our regional policy, an oncologic patient should receive HPN when meeting all the following criteria: (1) proven failure to meet nutrition requirements by the oral or enteral route, with impending risk of death due to malnutrition; (2) life expectancy $>2$ months; (3) Karnofsky performance status $\geq 50$; (4) control or absence of pain; (5) absence of severe organ dysfunctions; (6) written informed consent confirming that the patient will accept this modality of nutrition support; (7) approval by the physician responsible for HPN, by the oncologist, and by the general practitioner; (8) presence of environmental conditions compatible with HPN; (9) availability of a dedicated in-home caregiver; (10) and availability of a nursing team dedicated to the patient home care, as provided by the Regional Public Health Service. HPN was defined as patients receiving a PN bag at home on a 10- to 14-h per day basis.

Chemotherapy was defined as patients receiving intravenous or oral anticancer chemotherapeutic drugs. Chemotherapy regimens were administered by oncologists according to the patients' need (perioperative, adjuvant, or palliative chemotherapy) following the European Society for Medical Oncology (ESMO) Clinical Practice Guidelines (www.esmo.org). A detailed description of the chemotherapy regimens adopted in the different sites and stages of the tumors is beyond the aims of this paper. In those patients who were receiving HPN and intravenous chemotherapy, the PICC was used for both the therapies but never at the same time.

In all patients, maintenance/care of PICC was carried out by specifically trained nurses through home visits initially every day for 2-3 weeks and at least every 7 days thereafter, according to the recommendations of our regional health service. A strict policy of hand washing and of environmental hygiene was observed by nurses and caregivers at home. Similarly, an appropriate asepsis when managing the PICC; a strict policy for flushing the catheter with normal saline before and after use, with the pulsating "push/pause" plus positive-pressure method [27]; and the use of transparent dressings were adopted. The lines were also used for drawing blood and flushed with $20 \mathrm{ml}$ of normal saline post-blood draw. No heparin lock was used; PICCs were flushed with the pulsating push/pause plus positive-pressure method and were locked with 10-20 $\mathrm{ml}$ of normal saline [28]. 
Diagnosis of local infection and of CRBSI was carried out according to the guidelines issued by the Society for Healthcare Epidemiology of America-Infectious Diseases Society of America (IDSA) [29, 30]. Specifically, the clinical definition of CRBSI was a bacteremia or fungemia in a patient who has an intravascular device and more than one positive blood culture result obtained from the peripheral vein, clinical manifestations of infection, and no apparent source for bloodstream infection (BSI) (with the exception of the VAD), plus simultaneous quantitative cultures of blood with a ratio of $>3: 1$ colonyforming units/milliliter of blood (catheter vs. peripheral blood), or differential time to positivity (growth in a culture of blood obtained through a catheter hub is detected by an automated blood culture system at least $2 \mathrm{~h}$ earlier than a culture of simultaneously drawn peripheral blood of equal volume) [29].

Management of CRBSI (by removal and/or at least 1014 days of systemic antibiotic treatment plus antibiotic lock therapy, when recommended) closely followed the IDSA guidelines [30]. Catheter-related venous thrombosis was diagnosed and treated according to guidelines [2, 25, 26]; only symptomatic thrombosis was considered (i.e., local pain, edema, and signs suggesting thrombosis, later confirmed by ultrasound examination or Doppler technology). Mechanical complications were managed according to current guidelines [2, 27, 28].

Causes of PICC removal due to complications included local infection; CRBSI with indication for PICC removal because of failure of or contraindication to conservative treatment [30]; PICC-related venous thrombosis associated with catheter malfunction $[25,26]$; rupture of the external segment of the catheter, if impossible to repair; complete or partial $(>4 \mathrm{~cm})$ dislocation of the catheter; and lumen occlusion resistant to clearance techniques/treatments.

Statistical analysis

The duration of PICCs was expressed as median (range). The rates of complications were expressed per 1,000 catheter days (incidence rate) and/or as a percentage of total PICCs. Complication rates were compared using Fisher's exact or $\chi^{2}$ tests adjusted for catheter days. The level of significance was defined as a $P$ value $<0.05$. All analyses were carried out using SPSS 17.0 (SPSS, Inc., an IBM Company, Chicago, IL). For the analysis, each PICC placement was counted as a new event. This case series included the reported PICCs in our previous study [31].

\section{Results}

Since June 1, 2008, through May 31, 2013, 250 nonhospitalized adult cancer patients (98\% with solid malignancies, mainly gastrointestinal tumors) were consecutively enrolled in this study (Table 1). In $93 \%$ of cases, the patients were in stage III or IV according to TNM. One hundred seventy-seven patients (71\%) received chemotherapy during the study period. All patients received HPN, 213 (85\%) for more than $90 \%$ of the PICC life span. All of them were followed up until PICC removal or until a patient deceased and no one was lost at follow-up.

Two hundred sixty-nine PICCs were studied, for a total of 55,293 catheter days (median dwell time 184 days). With respect to device characteristics, 226 were $4 \mathrm{Fr}(84 \%)$ and 108 (40 \%) were power-injectable PICCs. PICCs were most commonly inserted in the right arm $(210 ; 78 \%)$, in the basilic vein $(191 ; 71 \%)$, and by nurses $(199 ; 74 \%)$.

Complications and outcomes are shown in Table 2. The incidence of CRBSIs was low ( 0.05 per 1,000 catheter days), mechanical complications were uncommon (13.1\%; 0.63 per 1,000 catheter days), and PICC-related symptomatic thrombosis was rare $(1.1 \% ; 0.05$ per 1,000 catheter days). The three episodes of CRBSI occurred 22, 79, and 127 days after PICC insertion, while the three episodes of thrombosis occurred 9, 16 , and 21 days after PICC insertion. The overall complication rate was $17.5 \%$ (0.85 per 1,000 catheter days) and PICCs were removed because of complications in $7 \%$ of cases. The rate of complications was not significantly different between the three types of PICCs. Because of complications, no patients required hospitalization. With respect to microbiology, CRBSIs were caused by coagulase-negative staphylococci (two cases) and Escherichia coli (one case). In two cases of CRBSI, PICCs were removed.

During this period, seven cancer patients had a PICC in site for more than 2 years - with a total dwell time of 6,499 catheter days - with two PICCs lasting more than 3 years. Table 3 shows the main characteristics of these patients.

Table 1 Characteristics of the patient population

\begin{tabular}{rl}
\hline$N$ & 250 \\
Female gender, $n(\%)$ & $127(51)$ \\
Age (years), median (range) & $65(26-85)$ \\
Tumor site, $n(\%)$ & \\
Stomach & $74(30)$ \\
Pancreas/biliary system & $52(21)$ \\
Colon/rectum & $35(14)$ \\
Esophagus & $21(8)$ \\
Ovary & $17(7)$ \\
Others & $51(20)$ \\
Stage, $n(\%) \quad$ & $17(7)$ \\
II & $43(17)$ \\
IV & $190(76)$ \\
ECOG PS, median (range) & $1(0-2)$ \\
\hline
\end{tabular}

ECOG Eastern Cooperative Oncology Group, PS performance status 
Table 2 Complications of 269 peripherally inserted central catheters (PICCs)

\begin{tabular}{ll}
\hline Duration (day), median (range) & $184(15-1,384)$ \\
Infectious complications & \\
Local infection, $n$ & 6 \\
$n / 1,000$ catheter days & 0.11 \\
CRBSI, $n$ & 3 \\
$n / 1,000$ catheter days & 0.05 \\
Total, $n(\%)$ & $9(3.3)$ \\
Venous thrombosis, $n(\%)$ & $3(1.1)$ \\
$n / 1,000$ catheter days & 0.05 \\
Mechanical complications & \\
Catheter dislocation, $n(\%)$ & $19(7.1)$ \\
Rupture of external tract, $n(\%)$ & $4(1.5)$ \\
Lumen occlusion, $n(\%)$ & $12(4.5)$ \\
Total, $n(\%)$ & $35(13.1)$ \\
$n / 1,000$ catheter days & 0.63 \\
Overall complications, $n(\%)$ & $47(17.5)$ \\
$n / 1,000$ catheter days & 0.85 \\
Causes of removal, $n(\%)$ & \\
Catheter complications & $19(7)$ \\
End of IV therapy & $85(32)$ \\
Death & $165(61)$ \\
Removal ratio,$n(\%)$ & $19 / 47(40)$ \\
\hline
\end{tabular}

$C R B S I$ catheter-related bloodstream infection, $I V$ intravenous

${ }^{a}$ Ratio between number of removals because of catheter complications and number of total complications

\section{Discussion}

Cancer, HPN, and chemotherapy are recognized risk factors for the development of severe complications (i.e., CRBSI and thrombosis) and mechanical complications (i.e., lumen occlusion, dislocation) in patients with a central $\operatorname{VAD}[1,6,7$, 31-33]. The main finding of this study was that, if inserted and managed according to proper evidence-based protocols, PICCs can be safely used in cancer patients receiving chemotherapy and/or HPN, with a low incidence of CRBSI, thrombosis, and mechanical complications; a long catheter life span; and a low probability of catheter removal because of complications.

Based on a large and growing clinical experience, PICCs started to be used frequently in cancer patients $[3,15,17,18$, 23, 31, 34-39]. However, conflicting evidences on the rate of PICC-related complications were reported in literature in oncology settings. Walshe et al. in 2002 documented an overall complication rate of 10.9 per 1,000 catheter days in 351 patients (58\% outpatients) with 366 PICCs used for multiple purposes (10,562 catheter days) [17], but argued for continued PICC use in the cancer population. Cheong et al. in 2004, in a small-size retrospective study (17 patients, 27 PICCs used for chemotherapy), found an overall complication rate of $40.7 \%$ catheter days [23]. Actually, at the same institution 2 years after the introduction of proper strategies to reduce PICC complications, Yap et al. described in 73 similar patients with 88 PICCs a reduced overall complication rate of $15.9 \%$ or 2 per 1,000 catheter days $(P=0.006)$ [18]. Worth et al. in 2009 described a CRBSI rate of 6.6 and a thrombosis rate of 7.7 per 1,000 catheter days in oncohematological patients with 75 PICCs [36]. Differently, in a 2011 study regarding 807 PICCs used for chemotherapy or autologous stem cell procedures in 727 patients with solid and hematogenous tumors, Mollee et al. reported a rate of BSI (1.81 per 1,000 catheter days over 41,876 catheter days) and concluded suggesting the use of PICCs in such population [38]. In our study, the overall complication rate was $17.5 \%$, but just 0.85 per 1,000 catheter days due to the long median PICC duration (about 6 months); moreover, PICCs were removed because of complications only in $7 \%$ of cases.

The rate of central catheter-related complications has changed: CRBSIs, thrombosis, and mechanical complications are lower than those reported in the last 20 years. Nowadays, the goal of "near zero" CRBSI - the most feared complication-is no longer a dream [40, 41]. In recent years, several technological novelties have considerably improved the safety of PICCs (i.e., ultrasound-guided venipuncture of the upper midarm, novel materials, sutureless devices for catheter securement), whereas new policies have successfully decreased the overall risk of complications (well-defined "bundles" of evidence-based interventions, strict policies on hand washing, proper skin antisepsis, training of healthcare professionals, etc.) $[14,18,37]$. In oncology patients, Tian et al. reported that after helpful interventions in reducing complications, the overall PICC complication rate has decreased from 30 to $11 \%$ $(P=0.0004)$ [37]. Harnage in a medical center-adopting the multimodality "bundle" for infection prevention - has reported for a huge number of PICCs (i.e., 12,577) an incidence of zero BSI per 1,000 catheter days for a period of 7 years [14]. Recently, a prospective study - with a large proportion of oncologic patients enrolled - has reported zero CRBSI and zero thrombosis in HPN patients with 48 PICCs [15]. Similarly, a prospective study at our institution with a small number of PICCs and a limited follow-up (65 PICCs and 18 months, respectively) reported the same results in cancer patients [31].

A critical issue is the scenario where the PICC is used because the in-hospital setting is markedly different from the out-hospital one. Chopra et al. clearly demonstrated that PICCs are associated with a lower risk of BSI $(0.5 \%)$ than central venous catheters $(2.1 \%)$ in outpatients [42]. Conversely, contradictory data on the rate of PICC-related BSI were described for inpatients (mainly, intensive care unit patients) [16]. In this study, a low incidence of CRBSI was reported ( 0.05 per 1,000 catheter/days) in non-hospitalized cancer patients. The following key components of our PICC 
Table 3 Characteristics of patients with a PICC dwell time longer than 2 years

\begin{tabular}{|c|c|c|c|c|c|c|c|}
\hline & Case 1 & Case 2 & Case 3 & Case 4 & Case 5 & Case 6 & Case 7 \\
\hline Gender & Female & Female & Female & Male & Female & Female & Male \\
\hline $\operatorname{Age}^{\mathrm{a}}$ (years) & 65 & 58 & 49 & 53 & 60 & 63 & 56 \\
\hline Tumor site & Pancreas & Ovary & Stomach & Rectum & Oral cavity ${ }^{\mathrm{b}}$ & Stomach & Stomach \\
\hline Stage $^{a}$ & III & II & IV & II & II & II & III \\
\hline Karnofsky PS ${ }^{\mathrm{a}}$ & 70 & 70 & 70 & 80 & 80 & 80 & 70 \\
\hline $\mathrm{CRP}^{\mathrm{a}}, \mathrm{mg} / \mathrm{l}$ & 1.2 & 9.3 & 0.4 & 8.7 & 9.7 & 9.1 & 2.1 \\
\hline Albumin $^{\mathrm{a}}, \mathrm{g} / \mathrm{dl}$ & 4.6 & 3.1 & 4.2 & 4.3 & 4.7 & 3.6 & 3.7 \\
\hline Body mass index ${ }^{a}$ & 22.2 & 21.3 & 14.5 & 23.9 & 21.1 & 21.1 & 20.9 \\
\hline Hospital (days) ${ }^{\mathrm{c}}, n$ & 141 & 122 & 61 & 29 & 105 & 16 & 46 \\
\hline Operation $^{\mathrm{c}}, n$ & 2 & 1 & 0 & 1 & 5 & 0 & 0 \\
\hline Chemotherapy $^{\mathrm{c}}$, cycles & 6 & 12 & 12 & 3 & 2 & 4 & 12 \\
\hline Radiation therapy $^{\mathrm{c}}$ & Yes & No & No & Yes & Yes & No & No \\
\hline HPN days ${ }^{\mathrm{c}}, n$ & 639 & 724 & 791 & 586 & $1,268^{\mathrm{d}}$ & 1,139 & $788^{d}$ \\
\hline Catheter days, $n$ & 790 & 763 & 824 & 782 & $1,384^{\mathrm{d}}$ & 1,154 & $802^{\mathrm{d}}$ \\
\hline Catheter complications & No & No & No & No & No & $\mathrm{Yes}^{\mathrm{e}}$ & No \\
\hline PICC removal & Yes $^{\mathrm{f}}$ & Yes $^{\mathrm{f}}$ & Yes $^{\mathrm{f}}$ & $\mathrm{Yes}^{\mathrm{g}}$ & No & Yes $^{\mathrm{f}}$ & No \\
\hline
\end{tabular}

PICC peripherally inserted central catheter, $P S$ performance status, $C R P$ C-reactive protein, $H P N$ home parenteral nutrition

${ }^{\text {a }}$ At the time of PICC insertion

${ }^{\mathrm{b}}$ Percutaneous endoscopic and radiologic gastrostomy not feasible

${ }^{\mathrm{c}}$ After PICC insertion

d On February 28, 2014

${ }^{\mathrm{e}}$ Lumen occlusion due to clots occurred after 953 catheter days and was successfully treated by infusing urokinase

${ }^{f}$ Cause: death

${ }^{\mathrm{g}}$ Cause: end of HPN

management may as well have been the reason for this result: (a) the consistent use of ultrasound guidance and sutureless devices [31], (b) the exclusive use of single-lumen PICCs [16], and (c) the consistent use of maximal barrier precautions and skin antisepsis with $2 \%$ chlorhexidine [30, 37].

Earlier studies have reported risks of symptomatic catheterrelated thrombosis as high as $28 \%$, but more recent studies suggest a much lower incidence at $5 \%$ or less $[17,43,44]$. Lee et al. reported in a prospective study over 76,713 patient days (500 cancer patients, 444 VADs with $65 \%$ PICCs) that the incidence of symptomatic catheter-related thrombosis was $4.3 \%$ or 0.3 per 1,000 catheter days [44]. Chopra et al. have recently reported in a meta-analysis that PICCs were associated with a higher risk of deep vein thrombosis in cancer patients [45]. Nevertheless, Tian et al. in a study on cancer patients with 267 PICCs reported that the incidence of thrombosis has decreased from 2.9 to $0.61 \%$ using relatively simple and inexpensive interventions [37].

In this study, a low incidence of symptomatic PICC-related thrombosis was reported $(1.1 \% ; 0.05$ per 1,000 catheter days). This result was probably due to several factors: (a) the consistent use of ultrasound guidance for PICC placement, (b) the consistent choice of deep veins of the upper midarm (mainly, the basilic vein), (c) the systematic choice of a vein with an appropriate ratio between catheter diameter and vein diameter (i.e., 1:3 ratio), (d) the consistent use of single-lumen PICCs, (e) the consistent use of sutureless devices, (f) the prevalent use of a PICC of relatively small diameter (i.e., $4 \mathrm{Fr}$ ), and (g) the consistent control of the position of the catheter tip, with reposition of the catheter if the tip of the PICC was not at the appropriate location. Indeed, data from literature demonstrated that the risk of thrombosis decreased when PICCs were placed according to this decision-making $[31,43,45]$. Moreover, our study was not designed to investigate the incidence of asymptomatic thrombosis; thus, the incidence most likely would have been higher if our patients systematically had been explored using ultrasound.

On the whole, the reasons for discrepancies between reported rates of PICC-related complications are not clearly known, but may include advances in catheter materials, securement devices, and insertion technique; differences in patient populations (e.g., non- and cancer patients, in- and outpatients); and design (e.g., retrospective vs. prospective) and methodological limitations of some of the studies (e.g., earlier experiences, small-size samples, different definitions of complications). 
Anecdotally, in this paper was reported a case series of seven patients with a PICC in site for more than 2 years, with two PICCs lasting more than 3 years. Although these PICCs were used also in the hospital setting - with a higher risk of complications than the home setting [42] - as well as for HPN, chemotherapy, and drawing blood, no infectious complications or thrombosis occurred and the PICCs were not removed because of complications. Despite the very small number of cases, it seems that PICCs, when optimally managed, can be even successfully used for very long periods in cancer patients requiring a long-term vascular access.

In summary, we believe that three key elements played a pivotal role to reduce the overall rate of complications and prolong the PICC life span in our patient population: (a) the availability of a knowledgeable and experienced central venous access team; (b) the use of ultrasound-guided venipuncture; and (c) a proper patients' education and a specific caregivers' training, along with close monitoring by trained nurses at home.

\section{Strengths and limitations of the study}

To the best of our knowledge, this is the largest study reporting catheter-related complications in a case series of non-hospitalized cancer patients with 269 PICCs used for chemotherapy and/or HPN for a long period (5 years and over 55,000 catheter days). If compared with previous studies in this field, our study has some relevant and original features: (1) data were collected through a clinical study and not from a database, registry, or questionnaire; (2) it was a prospective study; (3) only cancer patients were enrolled; (4) only outpatients were enrolled; (5) most of the enrolled patients $(71 \%)$ were receiving chemotherapy during the course of the study; (6) all PICCs were inserted with the same evidence-based protocol and all patients received the same evidence-based protocol of maintenance/care at home; (7) the median dwell time for PICCs was very long (more than 6 months); and (8) no patient was lost at follow-up.

Our study presented several limitations. First, this was a single-center study carried out by teams with a wellestablished experience in PICC placement and home management, as well as a well-defined collaboration with the oncologists. Second, in this study, almost all patients with solid tumors were enrolled; therefore, our results may not be extended to all cancer patients (e.g., hematological malignancies). Third, only non-hospitalized cancer patients receiving chemotherapy and/or HPN-always assisted at home by trained caregivers and specifically trained nurses - were included. Therefore, because this is a small subset of cancer patients, our results may not be generalizable to inpatient populations or different outpatient settings (e.g., patients receiving chemotherapy alone). Fourth, patient-related factors, such as impingement on quality of life and cost of VAD maintenance, have not been explored in this study. Finally, this was an observational study, and a trial comparing PICCs with well-defined long-term VADs (i.e., tunneled catheters and ports) needs to be carried out to recommend the use of PICCs as long-term VADs in non-hospitalized cancer patients.

Despite several limitations, our study suggests that PICCs can be successfully utilized as safe and long-lasting VADs in non-hospitalized cancer patients recording a low and acceptable incidence of overall complications.

Conflict of interest The authors declare no conflicts of interest and no funding.

Authors' contributions PC developed the research question and study design; performed the data acquisition, analysis, and interpretation; and drafted and finalized the manuscript. CB and MP assisted with the study design, data analysis/interpretation, and manuscript drafting. $\mathrm{CG}, \mathrm{CD}$, $\mathrm{BM}$, and $\mathrm{ADF}$ contributed to the data acquisition and provided consultation for data analysis. All authors reviewed the final manuscript and gave approval for submission. The authors have full control of all primary data and agree to allow the journal to review their data if requested.

Open Access This article is distributed under the terms of the Creative Commons Attribution Noncommercial License which permits any noncommercial use, distribution, and reproduction in any medium, provided the original author(s) and the source are credited.

\section{References}

1. Gallieni M, Pittiruti M, Biffi R (2008) Vascular access in oncology patients. CA Cancer J Clin 58:323-346

2. Pittiruti M, Hamilton H, Biffi R, MacFie J, Pertkiewicz M (2009) ESPEN guidelines on parenteral nutrition: central venous catheters (access, care, diagnosis and therapy of complications). Clin Nutr 28: 365-377

3. Yamada R, Morita T, Yashiro E, Otani H, Amano K, Tei Y, Inoue S (2010) Patient-reported usefulness of peripherally inserted central venous catheters in terminally ill cancer patients. J Pain Symptom Manag 40:60-66

4. Loughran SC, Borzatta M (1995) Peripherally inserted central catheters: a report of 2506 catheter days. JPEN J Parenter Enteral Nutr 19: 133-136

5. Alhimyary A, Fernandez C, Picard M, Tierno K, Pignatone N, Chan HS, Malt R, Souba W (1996) Safety and efficacy of total parenteral nutrition delivered via a peripherally inserted central venous catheter. Nutr Clin Pract 11:199-203

6. Ng PK, Ault MJ, Ellrodt AG, Maldonado L (1997) Peripherally inserted central catheters in general medicine. Mayo Clin Proc 72:225-233

7. Tokars JI, Cookson ST, McArthur MA, Boyer CL, McGeer AJ, Jarvis WR (1999) Prospective evaluation of risk factors for bloodstream infection in patients receiving home infusion therapy. Ann Intern Med 131:340-347

8. Duerksen D, Papineau N, Siemens J, Yaffe C (1999) Peripherally inserted central catheters for parenteral nutrition: a comparison with centrally inserted catheters. JPEN J Parenter Enteral Nutr 23:85-89

9. Moureau N, Poole S, Murdock MA, Gray SM, Semba CP (2002) Central venous catheters in home infusion care: outcomes analysis in 50,470 patients. J Vasc Interv Radiol 13:1009-1016

10. Gorski LA, Czaplewski LM (2004) Peripherally inserted central catheters and midline catheters for the homecare nurse. J Infus Nurs 27:399-409 
11. DeLegge MH, Borak G, Moore N (2005) Central venous access in the home parenteral nutrition population-you PICC. JPEN J Parenter Enteral Nutr 29:425-428

12. Ryder M (2006) Evidence based practice in the management of vascular access devices for home parenteral nutrition therapy. JPEN J Parenter Enteral Nutr 30:82-93

13. Maki DG, Kluger DM, Crnich CJ (2006) The risk of bloodstream infection in adults with different intravascular devices: a systematic review of 200 published prospective studies. Mayo Clin Proc 81: $1159-1171$

14. Harnage S (2012) Seven years of zero central-line-associated bloodstream infections. Br J Nurs 21:S6-S8, S10-12

15. Botella-Carretero JI, Carrero C, Guerra E, Valbuena B, Arrieta F, Calañas A, Zamarrón I, Balsa JA, Vázquez C (2013) Role of peripherally inserted central catheters in home parenteral nutrition: a 5-year prospective study. JPEN J Parenter Enteral Nutr 37:544-549

16. Chopra V, Ratz D, Kuhn L, Lopus T, Chenoweth C, Krein S (2014) Peripherally inserted central catheter-associated bloodstream infections: prevalence, patterns and predictors. Am J Med. doi:10.1016/j. amjmed.2014.01.001

17. Walshe LJ, Malak SF, Eagan J, Sepkowitz KA (2002) Complication rates among cancer patients with peripherally inserted central catheters. J Clin Oncol 20:3276-3281

18. Yap YS, Karapetis C, Lerose S, Iyer S, Koczwara B (2006) Reducing the risk of peripherally inserted central catheter line complications in the oncology setting. Eur J Cancer Care (Engl) 15:342-347

19. Pittiruti M, Brutti A, Celentano D, Pomponi M, Biasucci DG, Annetta MG, Scoppettuolo G (2012) Clinical experience with power-injectable PICCs in intensive care patients. Crit Care 16:R21

20. Horattas MC, Trupiano J, Hopkins S, Pasini D, Martino C, Murty A (2001) Changing concepts in long-term central venous access: catheter selection and cost savings. Am J Infect Control 29:32-40

21. Robinson MK, Mogensen KM, Grudinskas GF, Kohler S, Jacobs DO (2005) Improved care and reduced costs for patients requiring peripherally inserted central catheters: the role of bedside ultrasound and a dedicated team. JPEN J Parenter Enteral Nutr 29:374-379

22. Orr ME (2002) The peripherally inserted central catheter: what are the current indications for its use? Nutr Clin Pract 17:99-104

23. Cheong K, Perry D, Karapetis C, Koczwara B (2004) High rate of complications associated with peripherally inserted central venous catheters in patients with solid tumours. Int Med J 34:234-238

24. Pittiruti M, La Greca A, Scoppettuolo G (2011) The electrocardiographic method for positioning the tip of central venous catheters. $\mathrm{J}$ Vasc Access 12:280-291

25. Campisi C, Biffi R, Pittiruti M (2007) GAVeCeLT committee for the consensus catheter-related central venous thrombosis - the development of a nationwide consensus paper in Italy. J Assoc Vasc Access $12: 38-46$

26. Debourdeau P, Kassab Chahmi D, Le Gal G, Kriegel I, Desruennes E, Douard MC, Elalamy I, Meyer G, Mismetti P, Pavic M, Scrobohaci ML, Lévesque H, Renaudin JM, Farge D, Working group of the SOR; French National Federation of Cancer Centers 2008 (2009) SOR guidelines for the prevention and treatment of thrombosis associated with central venous catheters in patients with cancer: report from the working group. Ann Oncol 20:1459-1471

27. Royal College of Nursing (RCN) (2010) I.V. Therapy forum: standards for infusion therapy. London: RCN www.rcn.org.uk. Accessed 28 February 2014

28. Infusion Nurses Society (2006) Infusion nursing standards of practice. J Infus Nurs 29(suppl 1):S1-S92

29. Mermel LA, Allon M, Bouza E, Craven DE, Flynn P, O'Grady NP, Raad II, Rijnders BJ, Sherertz RJ, Warren DK (2009) Clinical practice guidelines for the diagnosis and management of intravascular catheter-related infection: 2009 update by the Infectious Diseases Society of America. Clin Infect Dis 49:1-45
30. O'Grady NP, Alexander M, Burns LA, Dellinger EP, Garland J, Heard SO, Lipsett PA, Masur H, Mermel LA, Pearson ML, Raad II, Randolph AG, Rupp ME, Saint S, Healthcare Infection Control Practices Advisory Committee (2011) Guidelines for the prevention of intravascular catheter-related infections. Am J Infect Control 39(4 suppl 1):S1-S34

31. Cotogni P, Pittiruti M, Barbero C, Monge T, Palmo A, Boggio Bertinet D (2013) Catheter-related complications in cancer patients on home parenteral nutrition: a prospective study of over 51,000 catheter days. JPEN J Parenter Enteral Nutr 37: 375-383

32. Bozzetti F, Mariani L, Bertinet DB, Chiavenna G, Crose N, De Cicco M, Gigli G, Micklewright A, Moreno Villares JM, Orban A, Pertkiewicz M, Pironi L, Vilas MP, Prins F, Thul P (2002) Central venous catheter complications in 447 patients on home parenteral nutrition: an analysis of over 100.000 catheter days. Clin Nutr 21: 475-485

33. Beghetto MG, Victorino J, Teixeira L, de Azevedo MJ (2005) Parenteral nutrition as a risk factor for central venous catheterrelated infection. JPEN J Parenter Enteral Nutr 29:367-373

34. Raad I, Davis S, Becker M, Hohn D, Houston D, Umphrey J, Bodey GP (1993) Low infection rate and long durability of nontunneled silastic catheters. A safe and cost-effective alternative for long-term venous access. Arch Intern Med 153:1791-1796

35. Molloy D, Smith LN, Aitchison T (2008) Cytotoxic chemotherapy for incurable colorectal cancer: living with a PICC-line. J Clin Nurs 17:2398-2407

36. Worth LJ, Seymour JF, Slavin MA (2009) Infective and thrombotic complications of central venous catheters in patients with hematological malignancy: prospective evaluation of nontunneled devices. Support Care Cancer 17:811-818

37. Tian G, Zhu Y, Qi L, Guo F, Xu H (2010) Efficacy of multifaceted interventions in reducing complications of peripherally inserted central catheter in adult oncology patients. Support Care Cancer 18: $1293-1298$

38. Mollee $\mathrm{P}$, Jones $\mathrm{M}$, Stackelroth J, van Kuilenburg R, Joubert W, Faoagali J, Looke D, Harper J, Clements A (2011) Catheter-associated bloodstream infection incidence and risk factors in adults with cancer: a prospective cohort study. J Hosp Infect 78:26-30

39. Bellesi S, Chiusolo P, De Pascale G, Pittiruti M, Scoppettuolo G, Metafuni E, Giammarco S, Sorà F, Laurenti L, Leone G, Sica S (2013) Peripherally inserted central catheters (PICCs) in the management of oncohematological patients submitted to autologous stem cell transplantation. Support Care Cancer 21:531-535

40. Ruiz-Santana S, Saavedra P, León C (2012) "Near zero" catheterrelated bloodstream infections: turning dreams into reality. Crit Care Med 40:3083-3084

41. Secola R, Lewis MA, Pike N, Needleman J, Doering L (2012) "Targeting to zero" in pediatric oncology: a review of central venous catheter-related bloodstream infections. J Pediatr Oncol Nurs 29:14-27

42. Chopra V, O'Horo JC, Rogers MA, Maki DG, Safdar N (2013) The risk of bloodstream infection associated with peripherally inserted central catheters compared with central venous catheters in adults: a systematic review and meta-analysis. Infect Control Hosp Epidemiol 34:908-918

43. Meyer BM (2011) Managing peripherally inserted central catheter thrombosis risk: a guide for clinical best practice. J Assoc Vasc Access 16:144-147

44. Lee AY, Levine MN, Butler G, Webb C, Costantini L, Gu C, Julian JA (2006) Incidence, risk factors, and outcomes of catheter-related thrombosis in adult patients with cancer. J Clin Oncol 24:1404-1408

45. Chopra V, Anand S, Hickner A, Buist M, Rogers MA, Saint S, Flanders SA (2013) Risk of venous thromboembolism associated with peripherally inserted central catheters: a systematic review and meta-analysis. Lancet 382:311-325 